\title{
Metastatic Rectal Carcinoma with Long-Term Remission due to Modern Multimodality Treatment
}

\author{
Natalja Eigeliene ${ }^{a, b}$ Jatta Saarenheimo ${ }^{a, c} \quad$ Viktor Wichmann $^{a, b}$ \\ Pia Österlund ${ }^{\mathrm{d}, \mathrm{e}}$ Antti Jekunen ${ }^{\mathrm{a}} \mathrm{b}$ \\ aDepartment of Oncology, Vaasa Central Hospital, Vaasa, Finland; bUniversity of Turku, \\ Department of Oncology and Radiotherapy, Turku, Finland; 'Department of Pathology, Vaasa \\ Central Hospital, Vaasa, Finland; dDepartment of Oncology, Tampere University Hospital and \\ University of Tampere, Tampere, Finland; eDepartment of Oncology \& Pathology, Karolinska \\ Institutet and Karolinska Comprehensive, Cancer Centre, Stockholm, Sweden
}

Keywords
Chemotherapy $\cdot$ Liver resection · Metastatic colorectal cancer $\cdot$ Radiotherapy $\cdot$ Targeted agents

\begin{abstract}
In the era of personalized medicine, systemic treatment with chemotherapy in combination with targeted drugs, tailored according to RAS and BRAF status, has improved the survival of patients with metastatic colorectal cancer ( $\mathrm{mCRC}$ ), but curative resection of metastases provides the only chance of cure. Here, we present a 40-year-old male with rectal adenocarcinoma and multiple bilateral synchronous liver metastases who has achieved long-term remission with multimodal treatment without resection of all metastatic lesions. This case emphasizes the need of repeated multidisciplinary team assessments and change of treatment intent if extraordinary responses are seen. The initial therapy consisted of short-course radiotherapy and surgery of the primary tumor followed by oxaliplatin-based combination chemotherapy and panitumumab with disease control intent. A complete radiologic response in $>20$ liver metastases in segments II-VIII was obtained. A biopsy-verified relapse of 3 liver metastases occurred at 9 months of treatment pause. Subsequently, major liver resection of 8 lesions was performed (4 with adenocarcinoma and 4 with cicatrix showing the challenge of disappearing lesions), followed by 6 months of adjuvant-like therapy. No relapse in MRI, PET, or CT has been noted since liver resection 6 years ago. Comprehensive genomic profiling of the primary tumor and liver metastases had similar driver mutations representing a low level of gene alteration and low diversity, possibly explaining the exceptional treatment response.
\end{abstract}




\section{Introduction}

Colorectal cancer (CRC) is the fourth most common cancer and the second cause of cancer deaths worldwide [1]. The most common site of metastases in CRC is the liver [2]. Approximately, $15-30 \%$ of rectal cancer patients either have liver metastases at the time of diagnosis or develop liver metastases during the course of the disease, and approximately $70 \%$ of metastatic CRCs (mCRC) have liver metastases. Over the last decades, the treatment of mCRC has evolved with improved median overall survival (OS) [3, 4].

The standard treatment for mCRC involves chemotherapy based on fluoropyrimidines in combination with oxaliplatin and/or irinotecan. Cytotoxics are often combined and used sequentially [5]. A combination of chemotherapy with biologic agents targeting either vascular endothelial growth factor or epidermal growth factor receptor (EGFR) has become a part of routine, in the first-line therapy. These combinations have improved response rates, progression-free survival, and OS [3, 4, 6-8].

In selected patients, metastasectomy can be included in the treatment of mCRC; the resection of liver metastases in particular can improve relapse-free survival and OS and can offer a possibility for cure in more than one-third $[5,9,10]$. Treatment of the primary rectal cancer with synchronous, potentially resectable metastases is challenging [9]. The traditional way of treating the primary cancer first has been challenged, and multidisciplinary team assessment is mandatory, upfront in those cases where a liver-first approach might be a better option. Local therapy for advanced rectal primary tumors includes preoperative shortcourse radiotherapy to improve local control and in inoperable cases chemoradiation instead.

CRC, like any other type of cancer, is a complex disease with significant molecular differences that affect the carcinogenesis, prognosis, and choice and response of treatments. Large, deep-sequencing studies have revealed common genetic mutations that are involved in several signaling pathways (WNT, RAS-MAPK, PI3K, TGF- $\beta, P 53$, and DNA mismatch-repair pathways) and associated with the initiation and progression of CRCs $[11,12]$. Clinically, the most important biomarker is the presence or absence of $R A S$ mutations. Mutations in $R A S$ result in the downstream activation of the $R A S$-mitogen-activated protein kinase (MAPK) or $P I 3 K$ pathways, which is further connected to the resistance to anti-EGFR therapy [11]. Determination of KRAS, NRAS, and BRAF mutations and microsatellite instability status (MSI-H) is part of routine clinical practice at initiation of treatment for mCRC [5], where the prevalence of $K R A S$ is shown to be around 45\%, NRAS 5\%, BRAF 4-21\%, and MSI-H 3-7\% [13-15].

The use of panitumumab and cetuximab, anti-EGFR antibodies, has resulted in earlier tumor shrinkage and better responses for $R A S$ and $B R A F$ wild-type mCRC. Here, we present a case of metastatic rectal adenocarcinoma in a 40-year-old male who responded to panitumumab and oxaliplatin-based treatment in a clinically important manner, giving him a chance of cure with multimodality treatment.

\section{Case Report}

A 40-year-old man, who had hemorrhoids but was otherwise healthy with ECOG performance status 0 , contacted a physician due to frequent bowel movements and bloody stools. He had no family history of cancer. Colonoscopy was performed in September 2013, and a low rectal tumor was noted and biopsied, without further findings in the colon. Pelvic MRI showed a T3a low rectal tumor, and lymph nodes status was N2. Whole-body CT scan showed 2 nonspecific liver lesions, but MRI revealed $>10$ metastatic nodules of $9 \mathrm{~mm}$ in segments II, III, IV, VI, VII, and VIII. Tumor markers CEA and CA19-9 were within the reference range $(<4.7$ $\mu \mathrm{g} / \mathrm{L}$ and $<27 \mathrm{kU} / \mathrm{L}$, respectively).

\section{Karger'}


On local MDT assessment, a decision of a short-course preoperative radiotherapy $(5 \times 5$ Gy) with immediate surgery of the symptomatic primary followed by systemic therapy was made. The patient received the short-course preoperative radiotherapy after which a laparoscopy-assisted abdominoperineal resection was performed in October 2013. On postoperative day 7, acute ileus was noted, and an explorative laparotomy was performed, in which a hematoma was evacuated, and the intestines were decompressed. According to the pathological report, a pT2a $(3 \mathrm{~cm}$ ) adenocarcinoma grade 3 was noted with N2b (11 out of 12 lymph nodes positive) with good margins and complete mesorectal fascia. Lymphatic invasion was noted, but not perineural or vascular invasion. The stage was thus IVa, with unresectable multiple synchronous liver metastases.

After surgery, an MRI was performed in November 2013 revealing at least 23 metastases of $11 \mathrm{~mm}$ in segments II, III, IV, V, VI, VII, and VIII, without signs of intrahepatic cholestasis. Liver function tests were within the reference range for transaminases, bilirubin, and albumin, and elevated grade 1 for alkaline phosphatase. Hemoglobin, leukocytes, and thrombocytes were within the reference range, and C-reactive protein was $16 \mathrm{mg} / \mathrm{L}$, that is, mildly elevated. Liver metastases were unresectable according to centralized liver MDT, and systemic therapy was recommended. Disease control intent chemotherapy was commenced with 3 weekly CAPOX (capecitabine $1000 \mathrm{mg} / \mathrm{m}^{2}$ twice a day on days 1-15 and oxaliplatin $130 \mathrm{mg} / \mathrm{m}^{2}$ on day 1). Molecular testing for KRAS and NRAS was requested before chemotherapy initiation, and results were available within a month showing wild-type $R A S$ status (BRAF was not routinely tested in 2013); and 3 weekly panitumumab $9 \mathrm{mg} / \mathrm{kg}$ was added to CAPOX (as infusional 5-fluorouracil-FOLFOX was not used at the institution at that time). On the third infusion of oxaliplatin, an anaphylactic reaction, with seizures, muscle cramps, shortness of breath, and pain in the chest, occurred. In subsequent cycles, oxaliplatin was omitted, and no allergic reactions re-appeared. After 2 cycles in January 2014, a partial response was noted, and in March 2014, a CR in the >20 liver metastases was seen. Furthermore, 4 cycles as consolidation were given in July 2014 when treatment was paused. Intensive radiologic follow-up was continued 2-3 monthly with continued CR.

In March 2015, 9 months after pausing treatment, a relapse with 3 metastases of $14 \mathrm{~mm}$ in the liver was observed on MRI. CEA was elevated to $5.1 \mu \mathrm{g} / \mathrm{L}$ having been within the reference range before $(<4.7 \mu \mathrm{g} / \mathrm{L})$, butCA19-9 was still within the reference range $(<27 \mathrm{kU} / \mathrm{L})$. A liver biopsy confirmed adenocarcinoma of rectal origin. Re-challenge of 1 cycle of panitumumab-capecitabine was started as neoadjuvant. The patient was referred to liver MDT, and relapses were resectable.

In late April 2015, liver resection at the tertiary liver center at Helsinki University Hospital was performed, with nonanatomical liver resections of segments IVB, V, and VIII for 8 metastases visualized in perioperative ultrasound. Four metastases with adenocarcinoma were R0 resected with minimal margins of 3-20 mm and a vitality of $90-100 \%$, and 4 showed only fibrosis (cicatrix) in pathology.

Adjuvant-like treatment with panitumumab-capecitabine was given for 6 cycles up to September 2015. Positron emission tomography (PET/CT) was done in February 2016 without any signs of abnormal activity. Follow-up with alternating MRI and CT is still ongoing, and no signs of relapse have been observed with last follow-up in January 2021 (Fig. 1). CEA and CA19-9 have been within the reference range during follow-up. All the given treatments are presented in Table 1.

The genetic profiles of the original tumor and of the liver metastases (after first-line treatment with panitumumab, oxaliplatin, and fluoropyrimidine) at relapse 17 months later were analyzed with FoundationOne $\mathrm{CDx}^{\mathrm{TM}}$ (F1CDx), which is a next-generation sequencingbased in vitro diagnostic for tissue [11]. The results verified that both the primary tumor and liver metastases after treatment were still KRAS, NRAS, and BRAF wild type and thus eligible

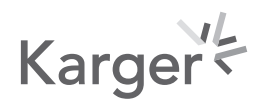



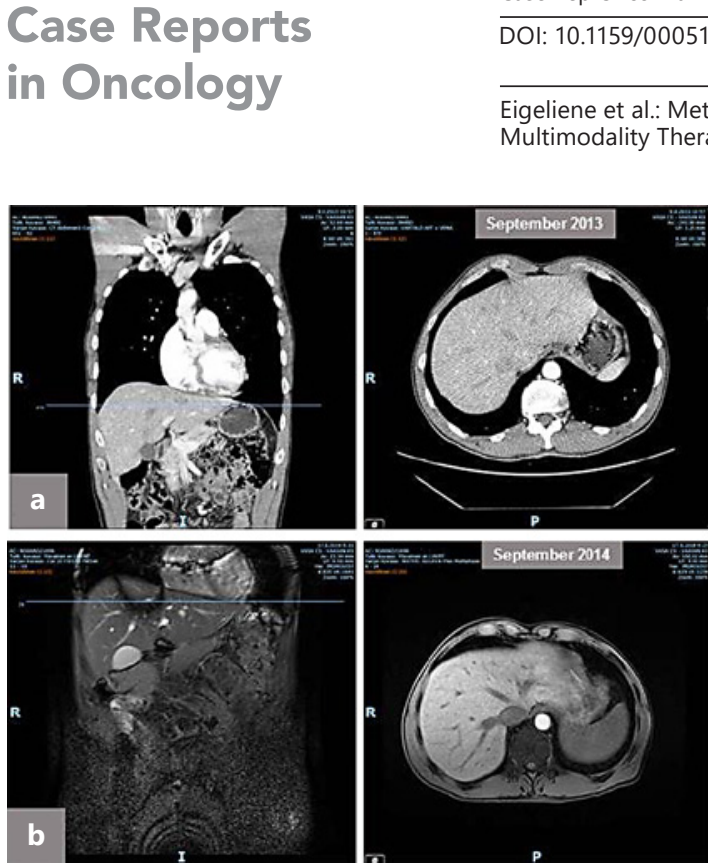
Multimodality Therapy

Eigeliene et al.: Metastatic Rectal Carcinoma Successfully Cured with Modern

Fig. 1. Abdominal scans demonstrating liver metastases. In September 2013, metastases were found in the liver, as shown in the CT scan (panel a). A complete radiologic response was noted in March 2015 as verified with MRI in September 2014 (panel b). In March 2015, relapse in the liver was noted on MRI (panel c). During follow-up in January 2021, there were no signs of metastasis in the liver according to the CT scan (panel d).

for panitumumab treatment. The most common mutations for secondary anti-EGFR resistance are emerging RAS mutations. Furthermore, none of the sporadic mutations, such as APC and TP53, found were clinically significant in the perspective of treatment decision or hereditary cancer, nor could they explain the exceptional treatment response, with long-term complete response in nonresected liver metastases. In addition, the patient was microsatellite stable and TMB-low (4 Muts/Mb), and thus had no Lynch syndrome, reflecting the chemotherapy-sensitive nature of the cancer. The mutations in the primary tumor and the metastases are presented in Table 2.

\section{Discussions}

This case demonstrates the effectiveness of using tailored treatment with systemic therapy in combination with radiotherapy and surgery of primary and liver metastases in an attempt to reach long-term remission and possibly cure. Here, of particular interest is the exceptional long-term CR to nonconventional capecitabine-based chemotherapy with an anti-EGFR antibody (panitumumab) and oxaliplatin until anaphylaxis, with a CR lasting $>5$ years. The COIN study shows higher responses, but no progression-free survival or OS benefit of adding cetuximab to CAPOX with low CR rates and short response duration [16]. Second, in the new-EPOC study, harm was noted when cetuximab was added to neoadjuvant and adjuvant CAPOX or FOLFOX in combination with liver resection [17]. This benefit on study level does not preclude exceptional responses in individuals. As well, it was found that approximately 1 of every 200 patients appears to have been potentially cured of mCRC by systemic therapy alone [18].

Recent insights into tumor heterogeneity allow a shift in the treatment paradigm predicting the response [19]. In this case, the primary tumor and liver metastases had similar driver mutations representing a low level of gene alteration and low diversity (only addition of $B R C A 2$ and $M Y C$ in liver metastases after treatment), possibly explaining the exceptional treatment response. 
Eigeliene et al.: Metastatic Rectal Carcinoma Successfully Cured with Modern Multimodality Therapy

Table 1. Details of the treatments given to the patient

\begin{tabular}{|c|c|c|}
\hline Month & Year & Treatment \\
\hline 10 & 2013 & Primary and synchronous liver metastases $\rightarrow$ MDT \\
\hline \multicolumn{3}{|c|}{ Local treatment } \\
\hline 10 & 2013 & Short-course radiotherapy $\rightarrow$ abdominoperineal resection \\
\hline 11 & 2013 & Verification and progression of 23 liver metastases \\
\hline
\end{tabular}

Disease control chemotherapy

11-12 2013 Capecitabine and oxaliplatin (CAPOX) x2, PR

12014

Panitumumab added to CAPOX x1

$1-7 \quad 2014$

Panitumumab and capecitabine x7, CR x2

Follow-up in CR

8 2014-2015 Regular follow-up with CT/MRI, clinical examination and

2 laboratory tests including tumor markers

Liver resection and adjuvant-like therapy

$\begin{array}{lll}3 & 2015 & \text { Biopsy-verified relapse of } 3 \text { liver metastases } \rightarrow \text { MDT } \\ 4 & 2015 & \text { Neoadjuvant panitumumab and capecitabine x1 } \\ 4 & 2015 & \text { Liver resection (8 lesions) } \rightarrow \text { MDT } \\ 5-9 & 2015 & \text { Adjuvant-like panitumumab and capecitabine x6 }\end{array}$

Follow-up after resection/CR

10 2015-2021 Regular follow-up with CT/MRI, clinical examination and

$1 \quad$ laboratory tests including tumor markers

CAPOX, capecitabine and oxaliplatin; MDT, Multidisciplinary Team assessment; PR, partial response according to RECIST 1.1; CR, complete response according to RECIST 1.1.

Table 2. Mutations found in the primary tumor and resected liver metastases

\begin{tabular}{ll}
\hline Primary tumor & Liver metastasis \\
\hline FBXW7 Q95* & FBXW7 Q95* \\
APC S1411 fs*4 & APC S1411 fs*4 \\
BCL2L1 amplification & BCL2L1 amplification \\
FAM123B R641* & FAM123B R641* \\
TP53 S313 fs*32 & TP53 S313 fs*32 \\
& BRCA2 F1427 fs*11 \\
MS-stable & MYC \\
TMB-low & MS-stable \\
\hline
\end{tabular}

There is no consensus on liver resection of disappearing lesions, but cure or longterm remission is rare $(17 \%)[9,20]$. With at least 23 lesions in all but segment $I$, the patient was unresectable initially, and resection of all regions with tumor initially was impossible. The exceptional response to systemic treatment enabled resection of re-occurring liver lesions. Here, a high vitality of $90-100 \%$ in the 4 metastases even after 1 cycle of neoadjuvant treatment was noted, with fibrosis in the rest of the resected ones, implicating 
impaired prognosis [21]. Thus, the patient would probably not have achieved a long-term remission with systemic therapy alone, without resection of the aggressive re-occurring lesions.

Intensive follow-up is very important in cases like this, and MRI is clearly better than CT in this case, as seen initially with 2 lesions on CT and $>20$ on MRI [22]. Tumor markers are not sensitive nor specific enough for detecting remaining cancer and are negative in $20-40 \%$ of cases, that is, this case has had marginally elevated CEA only once [23]. Confirmation of CR was obtained by following the patient for nearly 6 years after liver resection and for $>5$ years from stopping adjuvant therapy without any detectable signs of cancer. Thus, with long-term remission from multimodality treatment, the patient has a chance for cure.

The optimal use of the multimodality therapy arsenal in rectal cancer with synchronous metastases needs to be coordinated at MDTs at diagnosis and repeatedly during the treatment course. This is emphasized by this case in which tailored nonconventional treatments give long-term remission. The MDT assessments need clear processes and good timely logistics, especially between local hospitals and tertiary centers. This patient participated in the national multicenter MDT assessment study (RAXO), where repeated resectability assessment was centrally coordinated optimizing treatment delivery [24, 25].

\section{Conclusions}

We conclude that rectal cancer with liver metastasis can occasionally be cured with multimodality treatment. Molecular pathology should be performed upfront to guide treatment decisions. Larger genomic profiling can be performed on tumor tissue prior to any treatments and from a later time point after systemic therapy to tailor systemic treatment and exceptional re-challenge options according to the evidence of resistance.

\section{Statement of Ethics}

This study was conducted in accordance with Declaration of Helsinki and monitored independently. The protocol was approved by the Ethics Committee of Helsinki University Hospital (HUS242/13/February 03, 2011, and HUS/1288/2016), and the patient has given his oral and written informed consent for this case report.

\section{Conflict of Interest Statement}

The authors state that they have no conflicts of interest. They report research support for the RAXO study and institutional support for research from Amgen, Eli Lilly, Merck, Sanofi, Servier, and Roche (P.Ö. and A.J.). P.Ö. and A.J. have institutional support for research from Amgen, Eli Lilly, Merck, Sanofi, Servier, and Roche.

\section{Funding Sources}

This work was supported by the Competitive State Research Financing of the Expert Responsibility Area of TYKS (Turku University Hospital, Finland).

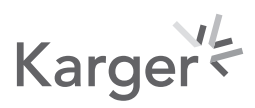




\section{Case Reports in Oncology}

\begin{tabular}{l|l}
\hline Case Rep Oncol 2021;14:1475-1482 \\
\hline DOI: 10.1159/000519044 & $\begin{array}{l}\text { @ 2021 The Author(s). Published by S. Karger AG, Basel } \\
\text { www.karger.com/cro }\end{array}$ \\
\hline
\end{tabular}

Eigeliene et al:: Metastatic Rectal Carcinoma Successfully Cured with Modern Multimodality Therapy

\section{Author Contributions}

Antti Jekunen contributed to concept and design. Natalja Eigeliene, Jatta Saarenheimo, and Viktor Wichmann contributed to acquisition, analysis, or interpretation of data. Natalja Eigeliene and Jatta Saarenheimo contributed to drafting of the manuscript. Pia Österlund and Antti Jekunen contributed to critical revision of the manuscript for important intellectual content. All authors read and approved the final manuscript.

\section{Data Availability Statement}

All data generated or analyzed during this study are included in this article. Further enquiries can be directed to the corresponding author.

\section{References}

1 Bray F, Ferlay J, Soerjomataram I, Siegel RL, Torre LA, Jemal A. Global cancer statistics 2018: GLOBOCAN estimates of incidence and mortality worldwide for 36 cancers in 185 countries. CA Cancer J Clin. 2018 Nov; 68(6): 394-424.

2 Cartwright TH. Treatment decisions after diagnosis of metastatic colorectal cancer. Clin Colorectal Cancer. 2012 Sep;11(3):155-66.

3 Elez E, Argilés G, Tabernero J. First-line treatment of metastatic colorectal cancer: interpreting FIRE-3, PEAK, and CALGB/SWOG 80405. Curr Treat Options Oncol. 2015 Nov;16(11):52.

4 Puccini A, Loupakis F, Stintzing S, Cao S, Battaglin F, Togunaka R, et al. Impact of polymorphisms within genes involved in regulating DNA methylation in patients with metastatic colorectal cancer enrolled in three independent, randomised, open-label clinical trials: a meta-analysis from TRIBE, MAVERICC and FIRE-3. Eur J Cancer. 2019 Apr;111:138-47.

5 Van Cutsem E, Cervantes A, Adam R, Sobrero A, Van Krieken JH, Aderka D, et al. ESMO consensus guidelines for the management of patients with metastatic colorectal cancer. Ann Oncol. 2016 Aug;27(8):1386-422.

6 Heinemann V, von Weikersthal LF, Decker T, Kiani A, Vehling-Kaiser U, Al-Batran SE, et al. FOLFIRI plus cetuximab versus FOLFIRI plus bevacizumab as first-line treatment for patients with metastatic colorectal cancer (FIRE-3): a randomised, open-label, phase 3 trial. Lancet Oncol. 2014 Sep;15(10):1065-75.

7 Cremolini C, Loupakis F, Antoniotti C, Lupi C, Sensi E, Lonardi S, et al. FOLFOXIRI plus bevacizumab versus FOLFIRI plus bevacizumab as first-line treatment of patients with metastatic colorectal cancer: updated overall survival and molecular subgroup analyses of the open-label, phase 3 TRIBE study. Lancet Oncol. 2015 Oct;16(13):1306-15.

8 Venook AP, Niedzwiecki D, Lenz HJ, Innocenti F, Fruth B, Meyerhardt JA, et al. Effect of first-line chemotherapy combined with cetuximab or bevacizumab on overall survival in patients with KRAS wild-type advanced or metastatic colorectal cancer: a randomized clinical trial. JAMA. 2017 Jun 20;317(23):2392-401.

9 Adam R, de Gramont A, Figueras J, Kokudo N, Kunstlinger F, Loyer E, et al. Managing synchronous liver metastases from colorectal cancer: a multidisciplinary international consensus. Cancer Treat Rev. 2015 Nov;41(9):729-41.

10 Modest DP, Rivera F, Bachet JB, de Braud F, Pietrantonio F, Koukakis R, et al. Panitumumab-based maintenance after oxaliplatin discontinuation in metastatic colorectal cancer: a retrospective analysis of two randomised trials. Int J Cancer. 2019 Jan 7;145(2):576-85.

11 Fearon ER. Molecular genetics of colorectal cancer. Annu Rev Pathol. 2011;6:479-507.

12 Cancer Genome Atlas N. Comprehensive molecular characterization of human colon and rectal cancer. Nature. 2012 Jul 18;487(7407):330-7.

13 Yamauchi M, Morikawa T, Kuchiba A, Imamura Y, Qian ZR, Nishihara R, et al. Assessment of colorectal cancer molecular features along bowel subsites challenges the conception of distinct dichotomy of proximal versus distal colorectum. Gut. 2012 Jun;61(6):847-54.

14 Arnold D, Lueza B, Douillard JY, Peeters M, Lenz HJ, Venook A, et al. Prognostic and predictive value of primary tumour side in patients with RAS wild-type metastatic colorectal cancer treated with chemotherapy and EGFR directed antibodies in six randomized trials. Ann Oncol. 2017 Aug 01;28(8):1713-29.

15 Nunes L, Aasebø K, Mathot L, Ljungström V, Edqvist PH, Sundström M, et al. Molecular characterization of a large unselected cohort of metastatic colorectal cancers in relation to primary tumor location, rare metastatic sites and prognosis. Acta Oncol. 2020 Apr;59(4):417-26.

16 Maughan TS, Adams RA, Smith CG, Meade AM, Seymour MT, Wilson RH, et al. Addition of cetuximab to oxaliplatin-based first-line combination chemotherapy for treatment of advanced colorectal cancer: results of the randomised phase 3 MRC COIN trial. Lancet. 2011 Jun 18;377(9783):2103-14. 
17 Bridgewater JA, Pugh SA, Maishman T, Eminton Z, Mellor J, Whitehead A, et al. Systemic chemotherapy with or without cetuximab in patients with resectable colorectal liver metastasis (New EPOC): long-term results of a multicentre, randomised, controlled, phase 3 trial. Lancet Oncol. 2020 Mar;21(3):398-411.

18 Dy GK, Hobday TJ, Nelson G, Windschitl HE, O'Connell MJ, Alberts SR, et al. Long-term survivors of metastatic colorectal cancer treated with systemic chemotherapy alone: a north central cancer treatment group review of 3811 patients, n0144. Clin Colorectal Cancer. 2009 Mar;8(2):88-93.

19 Sagaert X, Vanstapel A, Verbeek S. Tumor heterogeneity in colorectal cancer: what do we know so far? Pathobiology. 2018;85(1-2):72-84.

20 Benoist S, Brouquet A, Penna C, Julié C, El Hajjam M, Chagnon S, et al. Complete response of colorectal liver metastases after chemotherapy: does it mean cure? J Clin Oncol. 2006 Aug 20;24(24):3939-45.

21 Brouquet A, Zimmitti G, Kopetz S, Stift J, Julié C, Lemaistre AI, et al. Multicenter validation study of pathologic response and tumor thickness at the tumor-normal liver interface as independent predictors of disease-free survival after preoperative chemotherapy and surgery for colorectal liver metastases. Cancer. 2013 Aug 1; 119(15):2778-88.

22 Sivesgaard K, Larsen LP, Sørensen M, Kramer S, Schlander S, Amanavicius N, et al. Diagnostic accuracy of CE-CT, MRI and FDG PET/CT for detecting colorectal cancer liver metastases in patients considered eligible for hepatic resection and/or local ablation. Eur Radiol. 2018 Nov;28(11):4735-47.

23 Nicholson BD, Shinkins B, Pathiraja I, Roberts NW, James TJ, Mallett S, et al. Blood CEA levels for detecting recurrent colorectal cancer. Cochrane Database Syst Rev. 2015;(12):CD011134.

24 Isoniemi H, Uutela A, Nordin A, Lantto E, Kellokumpu I, Ovissi A, et al. Centralized repeated resectability assessment of patients with colorectal liver metastases during first-line treatment: prospective study. Br J Surg. 2021 Mar 22;108(7):817-25.

25 Osterlund P, Salminen T, Soveri L-M, Kallio R, Kellokumpu I, Lamminmäki A, et al. Repeated centralized multidisciplinary team assessment of resectability, clinical behavior, and outcomes in 1086 finnish metastatic colorectal cancer patients (RAXO): a nationwide prospective intervention study. The Lancet Regional Health Europe. 2021 Apr 01;3:100049. 